\title{
Análise de práticas de atenção ao parto e nascimento em maternidade pública
}

\author{
Analysis of childbirth care practices in a public maternity hospital
}

Maria Aline Rodrigues Barros ${ }^{1}$, Luana Silva de Sousa ${ }^{2}$, Camila Félix Américo ${ }^{1}$, Cinthia Maria Gomes da Costa Escoto Esteche ${ }^{1}$, Ana Kelve de Castro Damasceno ${ }^{1}$, Flávia Ximenes Vasconcelos ${ }^{1}$

Objetivo: analisar as práticas de atenção ao parto e nascimento em maternidade pública. Métodos: estudo transversal, descritivo, realizado em maternidade terciária, com 264 puérperas. Coleta procedida por meio de formulário, tendo como base as boas práticas de atenção ao parto. Resultados: prevalência das boas práticas de atenção ao parto. Observou-se que 233 (88,3\%) receberam informações, 256 (97,0\%) tiveram liberdade de posição, monitoramento fetal ocorreu em 260 (98,5\%), 198 (75,0\%) fizeram uso dos métodos não farmacológicos de alÍvio da dor, $263(99,6 \%)$ tiveram direito ao acompanhante, contato pele a pele em 258 (98,1\%). Conclusão: encontraram-se práticas, como amniotomia precoce e repetidos toques, que devem ser utilizadas com cautela. Ademais, verificou-se uso da manobra de Kristeller, embora em percentual baixo, a qual deve ser eliminada da assistência ao parto.

Descritores: Parto Natural; Parto Humanizado; Enfermagem Obstétrica.

Objective: to analyze childbirth and birth care practices in a public maternity hospital. Methods: a crosssectional descriptive study made in a tertiary maternity hospital with 264 puerperal women. Collection proceeded by means of a form based on good childbirth care practices. Results: prevalence of good childbirth care practices. It was observed that 233 (88.3\%) received information, $256(97.0 \%)$ had freedom of position, fetal monitoring occurred in 260 (98.5\%), 198 (75.0\%) used non-pharmacological pain relief methods, 263 (99.6\%) were entitled to a companion, skin-to-skin contact in 258 (98.1\%). Conclusion: practices such as early amniotomy and repeated touches were found that should be used with caution. In addition, the use of the Kristeller maneuver was verified, although in a low percentage, which should be eliminated from delivery care. Descriptors: Natural Childbirth; Humanizing Delivery; Obstetric Nursing.

${ }^{1}$ Universidade Federal do Ceará. Fortaleza, CE, Brasil.
${ }^{2}$ Universidade Estadual do Ceará. Fortaleza, CE, Brasil. 


\section{Introdução}

0 parto é um dos acontecimentos mais importante na vida da mulher, sendo os profissionais de saúde coadjuvantes desta experiência, cujo papel destes na assistência deve ser o de reconhecer e julgar a necessidade de intervenções no processo parturitivo, se utilizando de conhecimento, para que o binômio mãe e bebê tenham o bem-estar garantido ${ }^{(1)}$.

0 parto e o nascimento em ambiente hospitalar acontecem com a utilização de diversas tecnologias para torná-los mais seguros para mãe e bebê. Entretando, enquanto essas tecnologias contribuem para melhoria dos indicadores maternos e neonatais, por outro, validam modelo assistencial que lida com a gravidez, o parto e o nascimento como patologias e não como parte natural do ciclo de vida do ser humano ${ }^{(2)}$.

A Organização Mundial de Saúde, em 2018, divulgou recomendações sobre o cuidado intraparto, baseadas nas últimas evidências científicas. 0 objetivo é promover assistência de qualidade, independente da unidade de atendimento e nível de atenção à saúde ${ }^{(3)}$.

Para garantir a segurança do binômio materno-fetal durante o parto normal, a equipe de saúde deve estar munida de preparo e agir com base em evidências científicas durante o manejo de intercorrências e a realização de procedimentos. Além disso, é imprescindível a criação de vínculo entre profissional e cliente e assistência que considere a individualidade de cada mulher, para transmiti-la tranquilidade e confiança ${ }^{(4)}$.

Algumas instituições vêm implementando o trabalho integrado entre profissionais enfermeiros e médicos na assistência ao parto como políticas institucionais. Entretanto, por não se concretizar como prática sistematizada, a eficácia e a eficiência dessas práticas ainda não são conhecidas. A realização deste estudo, então, também se justifica com base na necessidade de produzir dados referentes a esse modelo assistencial $^{(5)}$.

A introdução de práticas baseadas em evidên- cias na assistência hospitalar perpassa por processo de superação de barreiras e mudanças de comportamentos e, por tal, exige mais do que conhecimento e convicções por parte dos profissionais ${ }^{(6)}$. Por isso, para que seja possível garantir o direito de decisão da gestante pela via de parto, de forma a considerar os ganhos e riscos em saúde, é necessário que a atenção à saúde aconteça de forma compartilhada entre a equipe multiprofissional e a mulher ${ }^{(2)}$.

O estudo dessas práticas de atenção ao parto e nascimento remete à necessidade de investigar e, de certa forma, acompanhar a implementação de boas práticas, para saber a real situação e em unidades de referência, e assim poder colaborar para análise crítica dessa realidade.

Frente ao exposto, este estudo objetivou analisar as práticas de atenção ao parto e nascimento em maternidade pública.

\section{Métodos}

Trata-se de estudo descritivo, de corte transversal e abordagem quantitativa. 0 campo eleito para pesquisa se constitui em maternidade de referência do estado do Ceará, Brasil. 0 período de coleta de dados ocorreu entre maio e outubro de 2017. A amostra probabilística foi representativa com base no número de partos ocorridos em 2016 para o cálculo de populações finitas, adotando-se intervalo de confiança de $95 \%$, prevalência de $50 \%$ para os casos de partos vaginais e erro amostral máximo permitido de 5\%, totalizando tamanho amostral de 264 mulheres. Os critérios de seleção foram: maioridade legal, gestação de risco habitual, em puerpério de parto normal e que apresentassem condições gerais que possibilitassem a participação na pesquisa enquanto respondente. Os critérios de exclusão foram mulheres que tinham diagnóstico de óbito fetal ou mortalidade neonatal precoce, devido à interferência na utilização de dados dos neonatos, bem como a não observação das práticas nestes casos. 
A coleta de dados aconteceu na enfermaria do puerpério, no primeiro andar da maternidade estudada, através da aplicação de formulário estruturado e pré-testado com 10 mulheres, por meio da técnica da entrevista, com tempo médio de 15 minutos para cada participante.

No primeiro momento, durante o internamento pós-parto, as puérperas foram contactadas para explanar sobre os objetivos do estudo, nos respectivos leitos, nas enfermarias de puerpério, pela pesquisadora. Em seguida, realizou-se entrevista individual com cada puérpera. 0 formulário foi elaborado pela pesquisadora e constituído por dados sociodemográficos e sobre a assistência durante o trabalho de parto e parto. As variáveis investigadas foram idade, estado civil, escolaridade, renda, informações recebidas na maternidade, privacidade respeitada, liberdade de posição, monitoramento fetal, métodos não farmacológicos de alívio da dor, presença do acompanhante, contato pele a pele, amniotomia, Kristeller, repetidos toques e episiotomia.

Os dados foram compilados e analisados através do programa estatístico Statistical Package for the Social Sciences, versão 20.0. As variáveis contínuas foram expressas mediante o cálculo da média; para as categóricas, obtiveram-se frequências absolutas e relativas. Para verificar possível associação entre variáveis, utilizaram-se dos testes Qui-quadrado e de Pearson e o Teste exato de Fisher, em que se estabeleceu o valor $\mathrm{p}<0,05$ como estatisticamente significativo. Entretanto, a aplicação de tais testes não revelou associações estatisticamente significantes. Os achados foram apresentados em tabelas e discutidos à luz da classificação das boas práticas da Organização Mundial da Saúde ${ }^{(3)}$.

A pesquisa atendeu às normas da Resolução 466/2012 que norteia pesquisas com seres humanos e obteve aprovação do Comitê de Ética em Pesquisa da Maternidade Escola Assis Chateaubriand, conforme Certificado de Apresentação para Apreciação Ética no 65798017.0.0000.5050 e parecer no 1.991.234/17.

\section{Resultados}

A caracterização sociodemográfica das puérperas permitiu inferir que 240 (90,9\%) possuíam menos de 35 anos; 205 (77,7\%) delas apresentaram união consensual, estável ou mediante casamento; 197 (74,6\%) renda mensal baixa, com valor menor ou igual a um salário mínimo; e quanto à escolaridade, (166) 62,9\% puérperas haviam concluído pelo menos o ensino fundamental.

Em relação à assistência ao parto, 150 (56,8\%) dos partos foram atendidos por médicos obstetras; $114(43,2 \%)$ por enfermeiras obstetras. Sobre o acesso à informação durante o atendimento na maternidade, $233(88,3 \%)$ das mulheres relataram que receberam orientações durante o trabalho de parto e parto pela equipe de plantão, sendo que 174 (74,6\%) obtiveram pela enfermeira, 25 (10,7\%) pelo médico, 11 (4,7\%) pelo técnico de enfermagem, 23 (9,8\%) não souberam informar a categoria profissional, isto pode ser devido ao fato de alguns profissionais não se identificarem e $31(11,7 \%)$ relataram não receber informações (Tabela 1$)$.

A privacidade foi respeitada em $251(95,1 \%)$ da população estudada. 0 monitoramento fetal foi relatado por 260 (98,5\%) da amostra. A presença de acompanhante foi respeitada pelos profissionais da instituição estudada em 263 (99,6\%) dos relatos. A utilização de métodos não farmacológicos para alívio da dor ao longo do trabalho de parto ocorreu em 75,0\% (198) das pacientes. A liberdade de posição foi referida por $256(97,0 \%)$ das mulheres, ademais a promoção do contato pele a pele ocorreu em $259(98,1 \%)$ das puérperas (Tabela 1).

Utilizou-se dos testes Qui-quadrado e de Pearson e o Teste exato de Fisher, em que se estabeleceu valor $\mathrm{p}<0,05$ como estatisticamente significativo. Entretanto, a aplicação de tais testes não revelou associações estatisticamente significantes. 
Tabela 1 - Distribuição das variáveis apropriadas durante a assistência ao trabalho de parto e nascimento $(n=264)$

\begin{tabular}{lc}
\hline Variáveis & n(\%) \\
\hline Informações & $233(88,3)$ \\
Sim & $31(11,7)$ \\
Não & \\
Privacidade & $251(95,1)$ \\
Sim & $31(4,9)$ \\
Não & \\
Liberdade de posição & $256(97)$ \\
Sim & $8(3)$ \\
Não & \\
Monitoramento fetal & $260(98,5)$ \\
Sim & $4(1,5)$ \\
Não & \\
Método não farmacológico & $198(75)$ \\
Sim & $66(25)$ \\
Não & \\
Presença do acompanhante & $259(98,1)$ \\
Sim & $5(1,9)$ \\
Não & \\
Contato pele a pele após o parto & $1(0,4)$ \\
Não & \\
\hline & \\
\hline
\end{tabular}

Tabela 2 - Distribuição das variáveis sobre as práticas não recomendadas durante o trabalho de parto e nascimento $(n=264)$

\begin{tabular}{lc}
\hline Variáveis & $\mathbf{n}(\%)$ \\
\hline Amniotomia & \\
Sim & $82(31,1)$ \\
Não & $182(68,9)$ \\
Kristeller & \\
Sim & $12(4,6)$ \\
Não & $252(95,5)$ \\
Episiotomia & \\
Sim & $15(5,7)$ \\
Não & $249(94,3)$ \\
Repetidos toques & \\
Sim & $147(55,7)$ \\
Não & $117(44,3)$ \\
\hline
\end{tabular}

Em relação às práticas que devem ser abolidas por serem claramente prejudiciais ou ineficazes, a tricotomia, a transferência da parturiente para outra sala no período expulsivo e a utilização de enema não foram relatadas pelas mulheres entrevistadas na instituição estudada, portanto, não foram ilustrados na Tabela 2.

No que se refere às práticas utilizadas de modo inapropriado durante o trabalho de parto e parto: a amniotomia foi realizada em 182 (68,9\%) das mulheres internadas com membranas íntegras; manobra de Kristeller, em 12 (4,6\%); episiotomia, em 15 (5,7\%); repetidos toques vaginais foram relatados por 147 $(55,7 \%)$ da população estudada durante o trabalho de parto.

\section{Discussão}

A limitação do estudo deve-se principalmente ao tipo, pois os estudos transversais têm como desvantagens a não relação direta de causa e efeito, bem como os fatores confundidores. Logo, seria interessante a realização de estudos que pudessem observar os atores envolvidos na assistência ao cuidado de mulheres durante o processo de parturição.

Os resultados deste estudo podem contribuir na mudança dos processos de saúde voltados para atenção à mulher, reconhecendo a importância de assistência que considere a singularidade de cada indivíduo. Busca-se o resgate da parturição como processo fisiológico, utilizando as atividades educativas e as boas práticas baseadas em evidências científicas, na evolução do trabalho de parto e parto.

Os dados do presente estudo evidenciaram menor número de partos de risco habitual atendidos somente por enfermeiras obstétricas na maternidade. Estudo corrobora com o impacto positivo da assistência ao parto prestada por enfermeiros obstetras e obstetrizes, como menos intervenções e maior satisfação das mulheres com a experiência do parto, e recomendam, assim, que gestores de saúde tomem parte em garantir condições favoráveis para maior atuação des- 
ses profissionais no processo de parturição $0^{(2)}$.

Sobre o acesso à informação durante o atendimento na maternidade, este foi satisfatório, pois mulheres relataram que receberam orientações durante o trabalho de parto e parto pela equipe de plantão. 0 acesso às informações de qualidade, como forma de empoderamento da mulher para promoção da participação ativa no parto, garante-lhe o papel de protagonista e torna a experiência da parturição processo consciente, o qual volta a ser percebido como natural e fisiológico ${ }^{(7)}$.

Os resultados revelaram que a maioria das participantes teve a privacidade preservada, corroborando com pesquisa realizada em maternidade pública de Curitiba, Brasil, em que 78,0\% das mulheres apresentaram intimidade conservada. Este é fator importante que exige a contribuição dos profissionais em garantila, pois a exposição indevida se opõe aos valores preconizados pela humanização e aos princípios de integralidade e individualidade $\mathrm{e}^{(8)}$.

Nos achados desta pesquisa, a liberdade para deambular e se movimentar foi semelhante a estudo recente, cuja taxa de deambulação foi de 208 (96,0\%) (5). A livre movimentação permite à parturiente adequar posturas confortáveis e adequadas ao período do trabalho de parto e parto. Isto deve ser estimulado por profissionais durante a assistência.

Esse fato demonstra como o serviço se encontra bem adequado a respeitar a implementação de boas práticas na assistência ao parto e nascimento, contribuindo para mudança real de cenário que até em então girava em torno dos profissionais e não das parturientes, sendo estas passivamente assistidas.

Com relação à monitorização fetal, observou-se, de forma rotineira, durante o trabalho de parto. A avaliação do bem-estar fetal em parturientes de baixo risco deve ser realizada com ausculta intermitente, em todos os locais de parto, o objetivo da monitoração do bem-estar fetal intraparto é avaliar a adequação da oxigenação fetal durante o trabalho de parto e, consequentemente, prevenir os danos resultantes da interrupção da transferência de oxigênio do ambiente para o feto, garantindo, assim, o nascimento de criança em boas condições ${ }^{(2)}$.

Quase todas as mulheres deste estudo permaneceram com acompanhante de livre escolha durante o período de internação. Isto converge com a Lei noo. 11.108/2005 no Brasil, em que a gestante tem direito a um acompanhante durante o trabalho de parto, parto e pós-parto imediato ${ }^{(9)}$.

A presença do acompanhante favorece experiência positiva no processo de parturição, pois garante maior satisfação e tranquilidade da mulher, aliviando o medo, a dor e a tensão ${ }^{(10)}$.

Nesse âmbito, destacam-se os métodos não farmacológicos para alívio da dor durante o trabalho de parto que, neste estudo, foram bem aceitos pelas mulheres. Isto reforça a necessidade de estabelecer estratégias de enfrentamento da dor pelos profissionais de saúde, a fim de promover assistência humanizada, em todo o processo de trabalho de parto e parto ${ }^{(11)}$.

Os dados revelaram que foi permitido o contato da puérperas com os filhos logo após o nascimento e, além disso, o aleitamento materno foi estimulado na primeira hora de vida do recém-nascido. Tudo isso corrobora com pesquisa realizada em maternidade de Pernambuco, Brasil, em que a maioria das mulheres recebeu o bebê imediatamente após o parto ${ }^{(12)}$. Esta prática deve ser estimulada independentemente da via de parto, pois favorece o sucesso do aleitamento materno exclusivo e o vínculo mãe-filho.

Em relação à amniotomia precoce, neste estudo, realizou-se de forma semelhante à outra pesquisa procedida em hospitais de nível terciário do Sistema de Saúde Pública da Andaluzia, Espanha, em que se observou em $34,3 \%$ das participantes do estudo ${ }^{(13)}$.

0 argumento utilizado para realização da amniotomia precoce é que diminuiria a duração do trabalho de parto. Entretanto, esse procedimento pode estar associado a algumas complicações potenciais, como aumento na ocorrência de desacelerações da frequência cardíaca fetal e infecção. Assim, os riscos e benefícios devem ser avaliados à luz das evidências científicas $^{(2)}$. Tal conduta, associada ou não à ocito- 
cina, não deve ser realizada rotineiramente em mulheres em trabalho de parto que estejam progredindo bem $^{(14)}$.

Com relação à realização de episiotomia, neste estudo, encontrou-se taxa abaixo de $30,0 \%$, recomendada pela Organização Mundial da Saúde. Outro estudo realizado em maternidade do Hospital Regional de São José, em Santa Catarina, Brasil, a prevalência de realização de episiotomia foi de $32,1 \%$, sendo maior em parturientes mais jovens e elevada escolarida$\mathrm{de}^{(15)}$.

O uso desnecessário da episiotomia causa risco de trauma perineal, necessidade de episiorrafia e complicações de cicatrização, além de reflexos diretos na vida sexual dessa mulher, sendo indicada se houver sinais de sofrimento fetal agudo, progressão insuficiente do parto ou lacerações mais graves ${ }^{(14)}$.

Dessa forma, os prestadores de cuidados obstétricos devem estar familiarizados e dispostos a considerar o uso de abordagens de baixa intervenção, quando apropriado, para o manejo intraparto de mulheres de baixo risco ${ }^{(16)}$.

Em relação à realização da manobra de Kristeller, houve baixa ocorrência neste estudo. Esta conduta é proscrita e considerada violação ao direito da mulher à integridade corporal, pois traz riscos para mãe - ruptura uterina - e bebê - sofrimento fetal.

Assim, estudiosos sinalizam a importância do envolvimento das instituições na reestruturação dos serviços, bem como no aprimoramento dos profissionais envolvidos, com base nas boas práticas de atenção ao parto e nascimento ${ }^{(5)}$.

No contexto brasileiro, pesquisa denominada Nascer no Brasil analisou 23.894 mulheres, de 191 municípios, em 266 hospitais entre públicos, privados e mistos, de médio e grande porte. E, dentre alguns resultados, o estudo demonstrou que a maioria das mulheres tem filhos por meio de cesarianas $(52,0 \%)$; no setor privado, esse número aumenta para $88,0 \% \mathrm{e}$ apenas 5,0\% dos partos vaginais foram sem intervenção. Em contrapartida, 43,1\% tiveram intervenções, dentre estas, parto com dieta zero $(74,8 \%)$, episiotomia (53,5\%), ocitocina sintética $(36,4 \%)$, manobra de Kristeller (36,1\%) e 34,1\% foram cesáreas eletivas ${ }^{(17)}$.

Em relação ao toque vaginal, esta pesquisa evidenciou quantitativo significativo. A Organização Mundial da Saúde propõe que esse exame seja realizado apenas quando necessário e com intervalo de quatro horas, durante o primeiro estágio do trabalho de parto $^{(2)}$. Entretanto, a maternidade estudada é campo de formação de profissionais da saúde, acarretando aumento da quantidade dos toques vaginais, embora não seja recomendado.

\section{Conclusão}

O estudo evidenciou que a maternidade pesquisada utilizava as boas práticas de atenção ao parto e nascimento, baseada nas políticas públicas de saúde e nas evidências científicas. No entanto, ainda foram encontradas práticas desnecessárias, como amniotomia precoce, repetidos toques e manobra de Kristeller, embora em percentual baixo, mas que devem ser evitados durante a assistência ao processo de parturição.

\section{Colaborações}

Barros MAR e Sousa LS contribuíram na concepção do projeto. Américo CF (in memoriam) colaborou com concepção do projeto. Esteche CMGCE, Damasceno AKC e Vasconcelos FX auxiliaram na análise e interpretação dos dados. Todos os autores cooperaram com redação do artigo, revisão crítica relevante do conteúdo intelectual e aprovação final da versão a ser publicada.

\section{Referências}

1. Ministério da Saúde (BR). Caderno Humaniza SUS. Humanização do parto e do nascimento [Internet]. 2014 [citado 2019 out 13]. Disponível em: http:// portaldeboaspraticas.iff.fiocruz.br/biblioteca/ humanizacao-do-parto-e-do-nascimento/ 
2. Ministério da Saúde (BR). Diretriz Nacional de Assistência ao Parto Normal - Conitec [Internet]. 2016 [citado 2019 out 13]. Disponível em: http://conitec.gov.br/images/Consultas/2016/ Relatorio_Diretriz-PartoNormal_CP.pdf

3. World Health Organization. World Health Organization recommendations: intrapartum care for a positive childbirth experience [Internet] 2018 [cited Oct 13, 2019]. Available from: https:// www.who.int/reproductivehealth/publications/ intrapartum-care-guidelines/en/

4. Pereira SS, Oliveira ICMS, Santos JBS, Carvalho MCMP. Natural childbirth: the work of nurses in the face of humanized care. Tempus Actas Saúde Coletiva. 2016; 10(3):199-213. doi: http://dx.doi. org/10.18569/tempus.v10i3.1727

5. Sousa AMM, Souza KV, Rezende EM, Martins DC, Lansky S. Practices in childbirth care in maternity with inclusion of obstetric nurses in Belo Horizonte, Minas Gerais. Esc Anna Nery. 2016; 20(2):32431. doi: 10.5935/1414-8145.20160044

6. Côrtes CT, Santos CS, Caroci AS, Oliveira SG, Oliveira SMJV, Riesco ML. Implementation methodology of practices based on scientific evidence for assistance in natural delivery: a pilot study. Rev Esc Enferm USP. 2015; 49(5):716-25. doi: http://dx.doi. org/10.1590/S0080-623420150000500002

7. Silva ALS, Nascimento ER, Coelho EAC. Nurses practices to promote dignity, participation and empowerment of women in natural childbirth. Esc Anna Nery. 2015; 19(3):424-31. doi: http:// dx.doi.org/10.5935/1414-8145.20150056

8. Apolinário D, Rabelo M, Wolff LDG, Souza SRRK, Leal GCG. Practices in delivery and birth care from mothers' perspective. Rev Rene. 2016; 7(1):208. doi: https://dx.doi.org/10.15253/21756783.2016000100004

9. Ministério da Saúde (BR). Lei n 11.108, de 7 de abril de 2005. Altera a Lei $n$ o 8.080, de 19 de setembro de 1990, para garantir as parturientes o direito à presença de acompanhante durante o trabalho de parto, parto e pós-parto imediato, no âmbito do Sistema Único de Saúde - SUS [Internet]. 2005 [citado 2019 fev. 22]. Disponivel em: http://www. planalto.gov.br/ccivil_03/_ato2004-2006/2005/ lei/l11108.htm
10. Rocha FAA, Fontenele FMC, Carvalho IR, Rodrigues IDCV, Sousa RA, Ferreira Júnior AR. Care during labor and birth: mothers' perception. Rev Rene. 2015; 16(6):782-9. doi: https://dx.doi. org/10.15253/2175-6783.2015000600003

11. Dias EG, Ferreira ARM, Martins AMC, Jesus MM, Alves JCS. Eficiência de métodos não farmacológicos para alívio da dor no trabalho de parto normal. Enferm Foco. 2018; 9(2):35-39. doi: https://doi. org/10.21675/2357-707X.2018.v9.n2.1398

12. Andrade PON, Silva JQP, Diniz CMM, Caminha MFC. Factors associated with obstetric abuse in vaginal birth care at a high-complexity maternity unit in Recife. Rev Bras Saúde Matern Infant. 2016; 16(1):29-37. doi: https://doi.org/dx.doi. org/10.1590/1806-93042016000100004

13. Hidalgo-Lopezosa P, Hidalgo-Maestre M, Rodríguez-Borrego MA. Birth plan compliance and its relation to maternal and neonatal outcomes. Rev Latino-Am Enfermagem. 2017; 25:e2953. doi:https://doi.org/dx.doi.org/10.1590/15188345.2007.2953

14. Ministério da Saúde (BR). Secretaria de Ciência, Tecnologia e Insumos Estratégicos. Diretrizes nacionais de assistência ao parto normal: versão resumida [Internet]. 2017 [citado 2019 set 20]. Disponível em: http://bvsms.saude.gov.br/bvs/ publicacoes/diretrizes_nacionais_assistencia_ parto_normal.pdf

15. Nunes RD, Mapelli AV, Nazário NO, Traebert E, Seemann M, Traebert J. Avaliação dos fatores determinantes à realização da episiotomia no parto vaginal. Enferm Foco. 2019; 10(1):71-5. doi: https://doi.org/10.21675/2357-707X.2019. v10.n1.1399

16. Committee on Obstetric Practice, King TL, Wharton KR, Ecker JL, Wax JR. Committee opinion no. 687: approaches to limit intervention during labor and birth. Obstet Gynecol. 2017; 129(2):e20-8. doi: dx.doi.org/10.1097/AOG.0000000000001905

17. Leal MC, Pereira APE, Domingues RMSM, Theme Filha MM, Dias MAB, Pereira, MN, et al. Obstetric interventions during labor and childbirth in Brazilian low-risk women. Cad Saúde Pública. 2014; 30(Suppl1):17-32. doi: http://dx.doi. org/10.1590/0102-311X00151513 